\title{
Kreuder Abroad. Zur Identitätskonstruktion eines problematischen Erfolgsautors
}

\section{Kreuder Abroad. On the Identity Construction of a Questionable Star of German Literature}

Jörg Krappmann

\begin{abstract}
The aim of the study is to read the up to now neglected articles about and by Ernst Kreuder in the journal Books Abroad as the culmination point in the career of this author, who rose to become a successful writer in the post-war period, but is hardly noticed in recent literary studies. The reasons for this later collapse are the close intermeshing between the construction of authorship and identity as well as the success that caused a discrepancy between an understanding as an elitist loner and the pursuit of broad public impact.
\end{abstract}

\section{Keywords}

Ernst Kreuder; post-war German Literature; poetological discourses; authorship; identity construction; Group 47; Ernst Erich Noth 
Die 2015 in Oldenburg veranstaltete Konferenz Ästhetik und Ideologie 1945 fragte nach Wandlung oder Kontinuität poetologischer Paradigmen (Haberland 2017) bei Autoren und Autorinnen der deutschsprachigen Nachkriegsliteratur. Es ging also um eine genauere Bestimmung, inwieweit der politische Umbruch von 1945 die Schreibverfahren und narratologischen Konzepte beeinflusste und veränderte. Die Ergebnisse fielen bei den jeweiligen Autoren so unterschiedlich aus, dass Detlef Haberland in seinem einleitenden Aufsatz des Konferenzbandes letztlich für eine Literaturgeschichtsschreibung anhand von Einzelanalysen plädierte. Das richtete sich ausdrücklich nicht gegen die bisherige Literaturgeschichtsschreibung, sondern gegen die unkritische Übernahme dort notgedrungen vorgenommener Verallgemeinerungen bei der Analyse von Texten einzelner Akteure oder in die Bewertungen von individuellen Publikationsstrategien. So dringlich geboten die präzise Verortung des Einzeltextes in die kulturellen und soziopolitischen Kontexte seiner Entstehungszeit und die Aufdeckung der Rezeptionsprozesse erscheint, würde sie doch „zu einer Literaturgeschichte von gigantischer Komplexität führen (müssen), wie sie nur mittels eines Hologramms oder einer Laser-Speicherung als vielschichtiger Struktur zu realisieren wäre“ (Haberland 2017: 19f.). Anhand von Ernst Kreuder kann gezeigt werden, dass die Frage nach und die Entscheidung über Wandel oder Kontinuität auch in Einzelanalysen nichts von ihrer Komplexität einbüßt und auf den unterschiedlichen Ebenen der literarischen Produktion zu konträren Ergebnissen führen kann.

Mitte der 1930er Jahre stieß Ernst Kreuder auf die poetologischen Überlegungen Alfred Döblins, die er in einer eigenwilligen Interpretation fortan zur Grundlage für sein eigenes Schreiben machte. Der neue Stil ist bereits in einigen Kurzgeschichten nachweisbar, wenn auch die meisten davon zum reinen Gelderwerb in recht kurzer Zeit produziert wurden. Er findet dann eine konsequente Umsetzung in den Erzähltexten der direkten Nachkriegszeit und ändert sich bis zum Roman Hörensagen (1969) kaum. Nur der letzte 1973 postum erschienene Roman Der Mann im Bahnwärterhaus folgt nicht mehr diesem poetologischen Programm, da er als eine Art Kompendium des Lebenswerks mehr kompilativ strukturiert als narratologisch konzis aufgebaut ist. Das soll hier nicht nochmals nachgezeichnet werden, führte mich aber in Bezug auf die Frage nach den Veränderungen der poetologischen Paradigmen dazu, bei Kreuders Erzähltexten über den Einschnitt 1945 hinweg Kontinuität zu konstatieren (Krappmann 2017). Verlässt man jedoch die Ebene der Poetik und der textuellen Verfahren, verkehrt sich dieser Befund in sein Gegenteil, denn zweifellos war ,1945 - als Signatur und nicht als Zeitpunkt verstanden - der entscheidende Wendepunkt in der schriftstellerischen Karriere von Ernst Kreuder.

Kreuder, der bereits in den 1920er Jahren vereinzelt Kurzgeschichten und Feuilletons veröffentlicht hatte, trat 1932 in die Redaktion des Simplicissimus in München ein. Nach der Übernahme der Zeitschrift durch die Nationalsozialisten, die auch von Teilen der Mitarbeiter befürwortet wurde, zog er sich in die ,Provinz‘ zurück. Die dort günstigeren Lebenshaltungskosten ermöglichten ihm weiterhin ein Leben als freier Schriftsteller, das er durch den systematischen Versand von Kurzgeschichten an lokale und regionale Zeitungen finanzierte. Die dafür geprägte Eigenbezeichnung „BUTKU = Bau- und Transport von Kurzgeschichten“ (Rauer 2008a: 240) verweist in ihrer Ironie auf den 
literarischen Stellenwert, den er den eigenen Publikationen zumaß. Während er einige der surrealen Grotesken aus der Zeit vor 1933 noch bis in die 1960er Jahre wiederveröffentlichte, kam den literarischen Arbeiten im Dritten Reich, einschließlich der Sammlungen Die Nacht des Gefangenen (1939) und Das Haus mit den drei Bäumen (1944), kaum mehr als ökonomische Bedeutung zu: „Versuchte weiterhin meine Semmeln schreibend zu verdienen mit mehr oder minder dämlichen Kurzgeschichten, sogenannten Räuberpistolen für unsere mehr und mehr, gleichgeschalteten' Wochenblätter und Tageszeitungen" (Kreuder 1971: 196). Die Sammelbände wurden aber durchaus in regionalen und überregionalen Periodika besprochen, so dass Kreuder in beschränktem Maße als ,etablierter 'Autor anzusehen ist, der von seinen Arbeiten leben konnte, ohne allerdings im zeitgenössischen Literaturbetrieb sonderlich aufzufallen. ${ }^{1}$

Kreuder wurde im Oktober 1940 zur Wehrmacht eingezogen und geriet am Ende des Krieges in amerikanische Gefangenschaft. Die Entbehrungen im Lager und ihre traumatischen Folgen verarbeitete er in den Erzählungen Schwebender Weg und Die Geschichte durchs Fenster, die 1947 erstmals gemeinsam in Buchform erschienen, sowie in einem Exkurs im Roman Herein ohne anzuklopfen (1954). Zunächst stellte er jedoch die längere Erzählung Die Gesellschaft vom Dachboden fertig, die bereits 1946 erscheinen konnte. Was daraufhin einsetzte, ist mit „Durchbruch“ (Schulz 1992: 78; Rauer 2008b: 114) nur unzureichend beschrieben. Vielmehr handelte es sich um eine Erfolgsgeschichte, die wohl nur in der Ausnahmesituation des (nicht nur) kulturellen Vakuums der Nachkriegszeit möglich war. Für Alfred Andersch war Kreuders Roman „die erste große Hoffnung der deutschen Nachkriegsliteratur" (Andersch 1974: 38) und Hans Werner Richter entwikkelte an ihm seine Konzeption eines magischen Realismus, die er als passende Form für die Literatur im Interregnum (vgl. Richter 1947: 10f.) und als Bollwerk gegen drohende eskapistische Ausweichbewegungen der deutschen Nachkriegsgesellschaft bewertete. Das ist insoweit erstaunlich als Die Gesellschaft vom Dachboden in rezenten Studien gerade „als Beispiel eines problematischen Eskapismus, das heißt eines Ausweichens von der historischen Situation in eine diffuse Sphäre der geistigen Indifferenz" (Egyptien 2006: 45) angesehen wird. Der Erzähltext hat tatsächlich nichts gemein mit einer Kahlschlagoder Trümmerliteratur, weist dagegen einige Merkmale der Deutschen Kalligraphie (Hokke 1946: 9) auf, die Gustav R. Hocke kurz zuvor und ebenfalls in der Zeitschrift Der Ruf als untauglich für eine deutsche Literatur nach dem Krieg einstufte. Darauf wird noch zurückzukommen sein.

Zunächst aber soll die Erfolgsspur Kreuders ohne Ablenkung nachgezeichnet werden. Der ersten 5.000 Exemplare umfassenden Auflage von Die Gesellschaft vom Dachboden folgte bereits 1947 eine zweite gleichumfängliche Auflage. Die 46 Rezensionen (vgl. Rauer 2008b: 252), die binnen zwei Jahre nach Erscheinen vorlagen, waren überaus positiv (vgl. Puknus 1982: 2; vgl. Schulz 1992: 402f.). Auf ebensolche Resonanz stießen auch die in einem Band zusammengefassten Erzählungen Schwebender Weg und Die Geschichte durchs Fenster (1947). Die englische Fassung des Romans (The Attic Pretenders) war 1948 die erste übersetze „deutsche Nachkriegserzählung überhaupt“ (Puknus 1982:

1 Weder die Kurzgeschichten selbst noch die Rezensionen enthalten „explizit nationalsozialistisches Sprachund Gedankengut" (Rauer 2008b: 65). 
2). Übersetzungen in weitere Sprachen folgten. Der Erfolg ermöglichte Kreuder, ein umfangreiches Romanprojekt wieder anzugehen, das er bereits 1938 - ausgestattet mit einem Honorarvorschuss des Verlags Rowohlt (vgl. Rauer 2008b: 91) - begonnen, aber aufgrund der Einberufung abgebrochen hatte. Die Unauffindbaren erschienen schließlich 1948 bei Rowohlt, der 1953 die Gesellschaft vom Dachboden auch in die rororo-Taschenbuchreihe übernahm, mit einer Startauflage von 50.000 Exemplaren. Als der für Kreuders Schreiben so entscheidende Alfred Döblin 1949 daran ging, eine literarische Klasse der Mainzer Akademie der Wissenschaft und der Literatur aufzubauen, wird Kreuder Gründungsmitglied. Im selben Jahr tritt er dem PEN-Club bei und wenig später wird er auch in die Deutsche Akademie für Sprache und Dichtung aufgenommen, von der ihm 1953 als zweitem Autor nach Gottfried Benn der Büchner-Preis verliehen wird.

Diese Erfolgsgeschichte ist ein komprimierter Auszug aus den wissenschaftlichen Arbeiten zu Kreuder. Nicht Bestandteil dieser Geschichte ist dort Kreuders Tätigkeit für die amerikanische Literaturzeitschrift Books Abroad, die jedoch, wie ich im Folgenden zu zeigen versuche, in gewisser Weise den Höhe- und Endpunkt der Erfolgskurve bildet. Die Zeitschrift wurde 1927 an der Universität Oklahoma gegründet und entwickelte sich rasch zu dem führenden Rezensionsorgan für europäische und lateinamerikanische Literatur (mit gelegentlichen Besprechungen zur asiatischen Literatur) in den Vereinigten Staaten, an dem sich zunehmend Philologen anderer Universitäten und Wissenschaftler anderer Disziplinen beteiligten. ${ }^{2}$ Einen deutlichen Schub erhielt die Zeitschrift, als 1949 Ernst Erich Noth (1909- 1983) die Funktion des leitenden Redakteurs übernahm, da dieser auch thematische Beiträge integrierte, die nicht mehr an einzelne Neuerscheinungen gebunden waren, sowie Berichte in Auftrag gab, die jeweils die Tendenzen der letzten 25 Jahre einer Nationalliteratur zusammenfassen sollten.

Unter dem Pseudonym Ernst Erich Noth hatte Paul Krantz 1931 den lebensideologisch geprägten Zeitroman Die Mietskaserne veröffentlicht, in dem er die Orientierungslosigkeit der Jugend in der Weimarer Republik anprangerte. Den daraus entstehenden vitalistischen Krisen begegnet er hier und in späteren Erzähltexten mit „individualpsychologischen“ (Lindner 1994: 233) Lösungen und einem spezifisch neusachlichen Irrationalismus. Der Problematik des Heranwachsens in politisch unsicheren Zeiten widmete sich auch seine Dissertation (Die Gestalt des jungen Menschen im deutschen Roman der Nachkriegszeit Manuskript 1933; Buchausgabe 1971), deren Disputatio an der Frankfurter Universität von den Nationalsozialisten wegen des Engagements Noths in einer kommunistischen Studentenvereinigung untersagt wurde. ${ }^{3}$ Er ging daraufhin nach Frankreich ins Exil, wo er an mehreren Zeitschriften mitarbeitete und seine schriftstellerische Laufbahn nun in französischer Sprache fortsetzte. Nach dem Einmarsch deutscher Truppen in Frankreich geriet er kurzfristig in ein Internierungslager und lebte dann im Untergrund. 1941 erhielt Noth ein Visum für die USA. Bevor er 1949 die Redakteursstelle bei Books Abroad übernahm, leitete er sieben Jahre die deutsche Programmsektion des

2 Die Zeitschrift wurde 1977 in World Literature Today umbenannt und erscheint in ihrer heutigen Form sechsmal jährlich.

3 Die Dissertation wurde 1971 nachträglich anerkannt, so dass Noth bis 1980 an der Goethe-Universität lehren konnte. 
Senders NBC (zu den biographischen Angaben vgl. Meier 1999). Bereits in der ersten Nummer von Books Abroad, die Noth selbständig betreute, rezensierte er Kreuders Roman Die Unauffindbaren:

\begin{abstract}
„Seldom given to overstatement, and well aware of the fact that undiscriminating, inflationary praise of too many a merely decent book has rendered certain superlatives quite meaningless, this reviewer feels nevertheless compelled to state in blunt admiration that this is the best book poetically, the most significant spiritually, that has come out for post-war Germany." (Noth 1950: 33).
\end{abstract}

Jahrelang, so Noth weiter, sei ungeduldig der Autor erwartet worden, der die deutsche Literatur von dem Terror der NS-Zeit und der anschließenden konformistischen Phase erlösen würde, nun aber sei in Person Kreuders dieser Autor erschienen, dessen Lektüre zudem ein atemberaubendes, faszinierend intellektuelles Abenteuer ist. Die ohnehin überaus emphatische Beurteilung wird von Noth schließlich noch durch die literarischen Texte überboten, die er als Vergleichsmaßstab für Kreuders Roman heranzieht: „John Cowper Powys' Wolf Solent, Robert Musil's Mann ohne Eigenschaften and James Joyce's Finnegans Wake" (Noth 1950: 33.). Damit wird Kreuder in den Rang der Weltliteratur erhoben.

Aufschlussreich ist Noths Wertschätzung für Kreuder auch als Stimme in der Kontroverse zwischen Exilautoren und den Vertretern der Inneren Emigration. Der immerhin wegen kommunistischer Aktivitäten ins Exil getriebene Noth hat, anders als manch anderer Exilautor, offensichtlich weder politische noch moralische Ressentiments einen westdeutschen Autor als den Repräsentanten der neuen deutschen Literatur zu inthronisieren. ${ }^{4}$ Noch in seiner Monographie The Contemporary German Novel (1960) warnte Noth „vor einer mechanistischen Dichotomie zwischen Exilliteratur und Literatur des Dritten Reiches und unterstreicht die vielfach übersehene Bedeutung der Literatur der ,inneren Emigration“" (Strelka 1989: 735). Damit vertrat er keine innovative oder isolierte Meinung, sondern lag ganz auf der Linie der Zeitschrift, in der schon Ende der 1940er Jahre, vor dem Eintritt Noths in die Redaktion, Neuerscheinungen u. a. von Werner Bergengruen $^{5}$ (Müller 1947) und wiederholt Ernst Wiechert ${ }^{6}$ (Pusey III. 1947; Meyer 1948) sowie die von Karl O. Paetel 1946 in New York herausgegebene Edition Deutsche Innere Emigration (Lederer 1947) besprochen wurden. Die von Noth verfassten Besprechungen zu Thomas Mann, der die Kontroverse durch seinen offenen Brief Warum ich nicht nach Deutschland zurückgehe (1945) mit entfacht hatte, sind jedoch hinsichtlich Doktor Faustus

4 Die Einschätzung Noths wird - eingedenk zahlreicher inniger Freundschaften, die durch Exilaufenthalt oder Verstrickungen im NS-Regime zerstört wurden - auch nicht dadurch abgeschwächt, dass Noth mit der Dichtergruppierung der Animalisten um Kreuder und Carl Mumm bereits vor 1933 flüchtig bekannt war (vgl. Noth 2009: 217-219).

5 Z. B. Die Sultansrose und andere Erzählungen durch den an der University of Oklahoma lehrenden Philosophen Gustav Müller (Books Abroad 21, Nr. 4, 1947, S. 456).

6 Z. B. Der Totenwald durch den amerikanischen Germanisten William W. Pusey III. (Books Abroad 21, Nr. 1, 1947, S. 46-47 oder das Gedenkbuch zum 60. Geburtstag Bekenntnis zu Ernst Wiechert durch die 1934 aus Deutschland in die USA emigrierte Germanistin Elizabeth M. Mayer (Books Abroad 22, Nr. 2, 1948, S. 91). 
(Noth 1949) ambivalent und in Bezug auf die Gesamtausgabe der Joseph-Tetralogie sogar ausgesprochen kritisch (Noth 1949). ${ }^{7}$

In einem der beiden Nachrufe auf Ernst Erich Noth, die 1984 in Books Abroad erschienen, hebt Ivar Ivask, ein späterer Nachfolger auf den Posten des Chefredakteurs, zwei Innovationen Noths besonders hervor, die der Zeitschrift ein anderes Profil verliehen hätten. Zum einen die 1951 veranstaltete, mehrteilige Rundfrage What's wrong with the Nobel Prize?, zum anderen die Einführung einer Rubrik, in der jeweils die Tendenzen der letzten 25 Jahre einer ,Nationalliteratur "überblicksweise dargestellt und eingeordnet wurden (vgl. Ivask 1984: 14). Für beide Unternehmungen kam für Noth nur Kreuder infrage. Die Rundfrage beantwortet Kreuder, als einziger publizierter Vertreter der bundesrepublikanischen Literatur, überraschend neutral. Über viele der bisherigen Preisträger könne er sich wegen Unkenntnis kein Urteil anmaßen, aber er könne dem Komitee einige Autoren für zukünftige Entscheidungen empfehlen: „John Cowper Powys, Gilbert Keith Chesterton, Rainer Maria Rilke, Paul Valéry, Thomas Wolfe, Aldous Huxley, Alfred Döblin and most of all Hans Henny Jahnn“ (Kreuder 1951: 116).

\section{A Quarter Century of German Literature}

Wenn Ernst Kreuder A Quarter Century of German Literature, seine Bilanz der neueren deutschsprachigen Literatur, mit der Gründung der Künstlervereinigung der Animalisten (1928) beginnen lässt, der u. a. er selbst und sein Co-Autor Carl Mumm angehörten, dann ist das sicherlich auch Ausdruck eines durch die raschen Erfolge gewachsenen Selbstbewusstseins. Zudem fungiert diese Einführung gegenüber einem internationalen Publikum als Bestätigung der Teilhabe des Verfassers an den dargestellten kulturellen Prozessen. Die Zeit zwischen 1927 und 1933 wird als Zeit der kulturellen und literarischen Fülle dargestellt, in der in Deutschland auch durch die wachsende Bedeutung des Rundfunks erstmals alle Möglichkeiten vorhanden waren, eine einflussreiche kritische Intelligenz herauszubilden, wie sie in England und Frankreich bereits seit Längerem wirkte:

„A literary intelligence and an intelligent literature as politically and sociologically operative forces, an intelligentsia with persisted effect, both as to width and depth, that might have be able to checkmate the Nazi propaganda which exploited the shortment of memory, the lack of taste, and the resentment of the populace“ (Kreuder - Mumm 1954: 6).

Diese reale Chance allerdings wäre vertan worden, weil die mahnenden Stimmen zwar gehört, aber nicht ernst genommen wurden. Albert Schweitzer, Rudolf Steiner und Martin Buber, die einer zunehmenden Politisierung der Kultur Modelle der Verinnerlichung

7 Die Kritik entzündet sich vor allem an der Darstellung der Erotik in Manns Roman: „The transparent, almost morbid obscenity of these scenes is matched only by so stupefying a dialogue that the reviewer was attempted to check with the title page to make sure that the book was really the work of one of the world's foremost writers" (Noth 1949: 366). 
entgegenstellten, wären trotz einer zeitweise breiten Rezeption ebenso einsame Rufer in der Wüste (preacher in the desert) geblieben, wie die Schriftsteller, die frühzeitig auf die Gefahren hingewiesen hätten, die durch die Verführung der Jugend (Frank Thieß: Die Verdammten 1922), die Verelendung breiter Gesellschaftsschichten (Alfred Döblin: Berlin Alexanderplatz 1929) und zunehmenden Rassismus (Hans Henny Jahnn: Strassenecke 1931) den Entwurf einer von literarischer Intelligenz bestimmten Gesellschaft bedrohten. Da die Fülle (abundance) nicht genutzt wurde, traten ihre negativen Merkmale in Erscheinung. Sie degenerierte zur Überfülle (superabundance), in der herausragende Leistungen wie Albrecht Schaeffers Helianth, Musils Der Mann ohne Eigenschaften oder die Erzähltexte Kafkas, von der Masse mediokrer Literatur überdeckt, kaum wahrgenommen wurden. Nicht das Überangebot wird von den Verfassern jedoch für das Scheitern der ,Blütezeit ' der deutschen Literatur am Ende der Weimarer Republik verantwortlich gemacht, sondern die Unfähigkeit eines bornierten Literatur- und Kulturbetriebs rechtzeitig und entschieden den früh erkennbaren Symptomen entgegengetreten zu sein. ${ }^{8}$

Dieses Deutungsmuster ist bei Kreuder aber nicht nur Vergangenheits-, sondern zugleich Gegenwartsdiagnose. In Bezug auf die soziokulturellen Prozesse der Moderne gibt es für ihn keine Stunde Null, keinen Neuanfang, sondern eine kontinuierlich verlaufende Entwicklung. „Die Masse“ - so Kreuder in einer Diskussionsrunde mit Mumm und Döblin zu Beginn der 1950er Jahre - „erscheint mir heute noch verrohter als zu Hitlers Zeiten“ (zitiert nach Schulz 1992: 305). Die Kritik muss deswegen zwar an die jeweiligen Zeittendenzen angepasst, aber nicht grundsätzlich geändert werden. Die Programmpunkte des Manifests der Animalisten, die der synoptischen Analyse vorangestellt sind, behalten grundsätzlich ihre Gültigkeit, nicht nur für die Zeit der Weimarer Republik, sondern auch für die Bundesrepublik im Publikationsjahr 1954.

Diese Konstanz in Kreuders Kulturkritik wurde bereits mehrfach festgestellt. Dabei ist allerdings zu berücksichtigen, dass Kreuders ebenso vehemente wie fundamentale Kritik auf der in der Aufklärung formulierten „Leitdifferenz“ (Hubig 2010: 56) Kultur - Zivilisation beruht, die in der Zwischenkriegszeit die soziopolitischen und philosophischen Diskurse prägte. Bei Kreuder schreibt sich diese Differenz weitgehend von Heideggers Technikfeindlichkeit her. Kultur bildet bei Kreuder keineswegs einen Gegensatz zur Natur (vgl. Nicklaus 2001: 100f.), sondern Kultur und Natur stehen in Opposition zu Technisierung und Bürokratisierung als Merkmale der Zivilisation. Im Beitrag für Books Abroad kann auf die Unterscheidung von Kultur und Zivilisation jedoch nicht explizit rekurriert werden, da im Englischen die Begriffe synonym verwendet werden, civilization also nicht negativ konnotiert ist. Sie artikuliert sich aber durchgehend implizit in der Kategorisierung der literarischen Texte, die danach beurteilt werden, ob und inwieweit sie den diagnostizierten zivilisatorischen Verfallserscheinungen entgegenwirken. Da der Zivilisationsprozess als Kontinuum verstanden wird, bedarf es auch keiner Veränderung der Wertungskriterien. Kreuder sieht von Beginn des Untersuchungszeitraums an in der

8 "Oversaturation? Literary schools, 'isms' and ideologies, excessive book production, intrigues for recognition and lucrative positions - the cunning ones wrote more 'excitingly' than the scrupulous - while the philistines banded together and, long before the infamous Machtergreifung, shouted against modern art." (Kreuder - Mumm 1954: 7). 
deutschen Literatur drei Tendenzen am Werk, anhand derer er auch seinen Überblick gliedert. Das kann hier nicht im Einzelnen nachverfolgt werden, aber die verwendeten Oberbegriffe der Tendenzen „(Neo-) Expressionism, Constructivism, (Neo-) Verism“ (Kreuder - Mumm 1954: 12) verdeutlichen bereits an sich ihre Verwendbarkeit für die Literatur vor und nach dem Krieg.

Die Liste der von Kreuder für gut befundenen Literatur nach 1945 enthält kaum Überraschendes. Aus der Literatur der Inneren Emigration werden Ernst Wiechert und Werner Bergengruen herausgegriffen, von den im Exil konzipierten Romanen Thomas Manns Doktor Faustus und Franz Werfels Der Stern der Ungeborenen, die Kreuder als umfassende Rechtfertigungen seiner zivilisationskritischen Thesen begreift. Zentrale Ankerpunkte der deutschen Nachkriegsliteratur sind weiterhin die „great admonishers and achievers" (Kreuder - Mumm 1954: 14) Alfred Döblin und Hans Henny Jahnn sowie deren künstlerische Nachfolger: Arno Schmidt, Hans Erich Nossack, Luise Rinser, Horst Lange und natürlich Kreuder selbst. Außerhalb dieser Strömung werden Stefan Andres, Emil Belzner, Ernst Jünger, Hermann Kasack und Elisabeth Langgässer vorgestellt. Ihre wirkliche Bedeutung erhält diese Liste erst durch die Aufzählung derjenigen Akteure, die 1954 bereits (mehrfach) literarisch hervorgetreten waren und von Kreuder stillschweigend übergangen wurden: Wolfgang Borchert, Heinrich Böll, Paul Celan, Günter Eich, Gerd Gaiser, Albrecht Goes, Rudolf Hagelstange, Wolfgang Koeppen, Hermann Lenz, Nelly Sachs, Wolfdietrich Schnurre, Günther Weisenborn. ${ }^{9}$ Ausgelassen werden auch die deutschsprachige Literatur in der Schweiz, die österreichische Literatur (trotz Werfel) und weitgehend auch die Literatur des Exils (so fehlen u. a. Feuchtwanger, Remarque oder Zuckmayer). ${ }^{10}$ Bei den Vertretern der DDR-Literatur, namentlich Becher, Brecht, Seghers und Wolf, konstatiert Kreuder einen falsch verstandenen Verismus, der lediglich auf politische Agitation setze und deswegen die für eine progressiv-moderne Literatur notwendige magisch-symbolische Erfassung der Tatsachenwelt verfehle.

Es dürfte deutlich geworden sein, wie klein der Ausschnitt der deutschsprachigen Literatur ist, der hier einem internationalen Publikum als lesenswert empfohlen wird. Nur was dem poetologischen Programm, einer Synthese aus ,Expressionism“ und ,Magism", entspricht, wird anerkannt und einstweilen in Ermangelung eines besseren Begriffs als ,New-Romanticism' angesprochen. In direkter Ansprache verteidigt Kreuder dieses Konzept gegenüber dem Realismus (Verismus) der Gruppe 47, der seiner Einschätzung nach literarisch wertlos bleiben wird:

„How often and repeatedly have the realists and naturalists attacked with pathetic polemic the liberty and sovereignty of creative imagination! The old Naturalists, already, believed that an ,idealistic', ,romantic' or ,visionary' art was definitely surpassed in the ,age of technology'. We know better today ..." (Kreuder - Mumm 1954: 13).

9 Die demokratische Grundeinstellung der Literatur der DDR wird anerkannt, kann aber über das Politische hinaus keine literarischen Qualitäten entwickeln.

10 Ob dies auf Anweisung der Redaktion erfolgte, weil selbständige Artikel zu Österreich und der Schweiz geplant waren, ist nicht mehr nachweisbar. 
Kreuders Besserwisserei entbehrt allerdings nicht einer gewissen Substanz, denn im Gegensatz zu Fritz Martini, für den sich „die Züge der neuen Prosa [...] noch kaum erkennen“ (Martini 1958: 595) lassen, macht Kreuder doch immerhin die Tendenzen namhaft, die im weiteren literaturwissenschaftlichen Diskurs trotz einiger Präzisierungen bestätigt wurden. Überdies fehlte auch noch 1958 in der neunten Auflage der von Fritz Martini ständig überarbeiteten und vom Publikum rege nachgefragten Deutschen Literaturgeschichte von den Anfängen bis zur Gegenwart weiterhin jeglicher Hinweis auf eine Existenz der Gruppe 47, mit der sich Kreuder in seinem Beitrag immerhin ausführlicher auseinandersetzt. ${ }^{11}$ Die teils harsche Kritik von Kreuder an der Gruppe bezieht sich erst auf die Forderung nach einer (für seinen Geschmack allzu) realistischen Literatur, die späterhin auch von Hans Werner Richter und Andersch propagiert wurde. Im März 1947, also ein halbes Jahr vor der später als Gründungsmythos der Gruppe 47 stilisierten Zusammenkunft der Mitarbeiter der geplanten Zeitschrift Der Skorpion, verpflichtete Hans Werner Richter die deutsche Gegenwartsliteratur noch auf die Aufgabe, „in der unmittelbaren realistischen Aussage dennoch hinter der Wirklichkeit das Unwirkliche, hinter der Realität das Irrationale [...] sichtbar werden zu lassen“ (Richter 1947: 11). Das ist mit Kreuders Konzeption eines „New-Romanticism“ und mit seinen literarischen Arbeiten nach 1945, die es „dem Menschen ermöglichen, am Wirklichen zu partizipieren, an jenem Magischen, das auf geheime Weise Gegensätze vereint“ (Egyptien 2006: 45), durchaus kompatibel. Anderschs eingangs erwähnte Wertschätzung Kreuders ergibt sich eben weitgehend daraus, dass er die Forderung Richters durch Die Gesellschaft vom Dachboden eingelöst sah. Erst durch die gleichzeitige Förderung eines Magischen Realismus durch die ostdeutsche Literaturwissenschaft (vgl. Krappmann 2013: 530f.) und die unterschiedlichen literarischen Konzepte jüngerer Gruppenmitglieder wurde dieses Konzept in Richtung des von Kreuder angeprangerten Verismus später abgeändert. ${ }^{12}$ Auch in Anderschs Essay Deutsche Literatur der Entscheidung (1948), der mangels anderer theoretischer Äußerungen das unwidersprochene Programm der Gruppe 47 blieb, sind noch einige Gemeinsamkeiten vorhanden, etwa in der Bewertung Elisabeth Langgässers (vgl. den Nachruf Kreuders auf Langgässer [Kreuder 1950], der auch in englischer Übersetzung in Books Abroad erschien [Kreuder 1951]). Trotz der Anerkennung der christlichen Überzeugung der Autorin lehnte Andersch jedoch in seinem Essay die von Alfred Döblin (Die literarische Situation 1947) geforderte „katholische Erneuerung“ (Williams 1991: 33) der Nachkriegsliteratur entschieden ab. Damit zog er neben den differenten poetischen Vorstellungen die zweite Demarkationslinie zwischen der Gruppe 47 und Kreuder, der seinem Vorbild Döblin in die Mainzer Akademie folgte, die freilich zu diesem Zeitpunkt auch größeres Prestige versprach als die lose Gruppierung junger Autoren, deren spätere Machtfülle im Kulturbetrieb der Bundesrepublik noch nicht abzusehen war.

11 Erst die zehnte Auflage von 1960 konnte nach dem „Romanjahr 1959“ (Schalk 1997: 238) mit den Romanen von Böll, Grass und Johnson an der Gruppe 47 nicht mehr vorbeigehen.

12 Die doppelte Besetzung des Begriffs Magischer Realismus veranlaßte mglw. Kreuder auf den „dubious term New-Romanticism“ (Kreuder - Mumm 1954: 12) zurückzugreifen, der wiederum dazu führte, dass Kreuder als weltflüchtiger Romantiker von der Literaturkritik missverstanden wurde (vgl. Fiedler 1990: 620-634). 


\section{Absturz mit/aus Konsequenz}

Ernst Kreuder gehörte in der Nachkriegszeit und der frühen Bundesrepublik zu den big playern der deutschen Literatur. Das belegt die Wertschätzung durch so unterschiedliche Akteure des zeitgenössischen Kulturbetriebs wie Alfred Andersch, Alfred Döblin, Ernst Erich Noth noch mehr als die institutionelle Anerkennung in Form von Preisen oder die positiven Urteile der Literaturkritik. In den einschlägigen Literaturgeschichten ist heute davon nichts geblieben. Im fast 900 Seiten umfassenden dritten Band (19181980) der von Viktor Žmegač verantworteten Geschichte der deutschen Literatur vom 18. Jahrhundert bis zur Gegenwart (1984) wird Kreuder nicht erwähnt und in Horst A. Glasers Kompendium Deutsche Literatur zwischen 1945 und 1995 darf er nur einmal als Vertreter einer eskapistischen Literatur auftreten, um von Heinrich Böll kritisiert zu werden (vgl. Brenner 1997: 42). Obwohl die Liste unschwer verlängert werden könnte, sollen die beiden prominenten Beispiele genügen. Eine Ausnahme davon ist Jürgen Egyptien, der in seiner komprimierten Einführung Kreuder einigen Raum widmet (Egyptien 2006). Das ist symptomatisch. Denn es ist nicht so, dass Kreuder von der Literaturwissenschaft vollständig ignoriert würde, sondern er findet vielmehr, mit zeitlichen Abständen, aber doch immer wieder, Fürsprecher. Deren Faszination geht einerseits von der Aktualität der Themen in Kreuders Romanen und Erzählungen aus, andererseits fordert eben die Diskrepanz zwischen Erfolg und Vergessenheit dazu heraus, nach Gründen für den Absturz zu suchen.

Zuletzt begeisterte sich Manfred Windfuhr für Kreuders „Ökotadel“ (Windfuhr 2018: 464). Ihm gelinge es „die Hauptvorgänge seiner Zeit [...], die Kriegswirklichkeit und die Kennzeichen der Zivilisation, Mechanisierung und Termitisierung" anzusprechen und gleichzeitig "prognostische Orientierung“ anzubieten (Windfuhr 2018: 466), die in den gegenwärtigen ökologischen und gesellschaftspolitischen Debatten Wirkung entfalten könnte: „Es wird Zeit, Ernst Kreuder wiederzuentdecken“ (Windfuhr 2018: 468). Diesem Anliegen wird - so es auf eine größere Leserschaft zielt - abermals kein Erfolg beschieden sein, denn Kreuder entspricht dem Typus des Problematischen Autors, den Reiner Stach an dem mit Kreuder befreundeten und ideell verbundenen Hans Henny Jahnn entwickelte:

\footnotetext{
„Der Problematische Autor [...] tritt nackt auf die Bühne. Seine Obsessionen, seine Begierden, seine Vorurteile und Dummheiten, Ekel, Lust und Todesangst - alles hat er ausgebreitet und starrt dabei dem Leser unverwandt in die Augen. Dem wird dabei unbehaglich zumute. Er ist berührt, vielleicht sogar erschüttert, er verspürt eine kathartische Wirkung, aber irgendwann ist es genug. Er möchte selbst darüber entscheiden, wie tief er sich ergreifen läßt. Der problematische Autor läßt nicht locker; er ist wie der Bettler, der, anstatt sein bißchen Kunst auf irgendeinem kindischen Instrument zu üben, uns ständig die Geschichte seines vertanen Lebens aufdrängt, und wie dieser muß er bald erkennen, daß seine Geschäfte schlecht gehen.“ (Stach 1996: 349f.).
}

Die Eindringlichkeit und Offenheit, mit der dieser Typus sein Anliegen vorträgt, wird ihm rasch eine verschworene Fangemeinde schaffen, aber nicht mainstreamtauglich 
werden. Es kommt zu einer „Polarisierung zwischen wenigen Anhängern und vielen Gleichgültigen“ (Stach 1996: 360), die sich auch im Umgang der Literaturwissenschaft mit dem Autor Kreuder nachweisen lässt. Da die Bedingungen für den Misserfolg also in der internen Identitätskonstruktion des Autors liegen, streifen externe Erklärungsversuche - Kreuder sei „ein Opfer der Gruppe 47“ (Deschner 1973: 42) oder wäre von der Literaturkritik falsch etikettiert worden (vgl. Fiedler 1990: 621) - nur die Peripherie des eigentlichen Problems.

In Kreuders Texten ist seit den ersten Publikationen nach 1945 eine insistierende Didaxe fest verankert, die in Form von monologischen Predigten die Diegese unterbricht. Seine Protagonisten werden dann zum Sprachrohr des Autors, die mit dichterpriesterlicher Attitüde die Ansichten Kreuders über die Literatur und die Welt verkündigen. Dass er diese „frühgrünen Predigten“ (Rauer) in Narrative unterhaltender Genres vom Märchen bis zum Kriminal- und Spionageroman kleidet, ist Teil seiner poetologischen Strategie: Die ungewöhnliche Welterkenntnis soll dadurch einer größeren Gemeinde vermittelt und deren Blick wieder auf das Eigentliche ausgerichtet werden (vgl. Krappmann 2013: 293-295). Eng damit verbunden ist die Struktur des verschworenen und oft auch verschwörerischen Geheimbundes, die die Figurenkonstellation aller Romane und Erzählungen Kreuders bestimmt. Stets werden eine oder mehrere männliche Figuren in das Geheimnis der magisch-realistischen Weltsicht eingeführt. Der Leser vollzieht den Einweihungsprozess des (oder der) Protagonisten nach und wird gleichsam mitinitiiert.

Das Selbstverständnis Kreuders ist dabei das einer elitärkulturellen, quasi-religiösen Gesandtschaft und die rasch aufeinanderfolgenden Ehrungen und Anerkennungen überzeugen ihn davon, kein ,preacher in the desert ${ }^{`}$ zu sein. Das gestiegene Selbstbewusstsein kulminiert schließlich 1954 in zweifacher Form. Zum einen durch die Erzählerfigur „Ernst Kreuder“ im Roman Herein ohne anzuklopfen. Statt der bisherigen figuralen Sprachrohre tritt er nun als Sprecher seiner selbst auf, um ein Kultur- und Literaturverständnis zu präsentieren, das - einschließlich der propagierten oder abgelehnten Schriftstellerkollegen - dem aus A Quarter Century of German Literature gleicht. Zum anderen zeigt das die Struktur eben dieses Beitrags für Books Abroad. Die Autorposition, die hier eingenommen wird, entspricht dem rasanten Aufstieg Kreuders in den vorangegangenen sieben Jahren und der weltliterarischen Nobilitierung durch Ernst Erich Noth. An zentralen Textpositionen setzt sich Kreuder selbst in Szene. Als Mitautor des animalistischen Manifests liefert er am Anfang des Beitrags das Erklärungsmoment für die Katastrophe des Dritten Reiches. In der Mitte präsentiert er seine Erzähltexte nach 1945 als die zentralen Dokumente des New-Romanticism, und auch der letzte Satz, der die Zukunft der deutschen Literatur vorausdeutet, ist ihm vorbehalten: „In his forthcoming novel Herein ohne anzuklopfen Ernst Kreuder attemps a synthesis of modern expressionistic art, existentialist radicalism and veristic veracity" (Kreuder - Mumm 1954: 14).

Nachdem dieser Roman - mit Stach gesprochen - nicht die Zahl derer sinken ließ, die den zivilisationskritischen, poetologischen und weltanschaulichen Thesen Kreuders gleichgültig gegenüberstanden, zeigte sich die Brüchigkeit seiner Identitätskonstruktion, die auf der Überzeugung aufbaute, eine „Existenz von Rang“ (Rauer 2008b: 331) zu sein. $\mathrm{Zu}$ Recht verweist Stephan Rauer darauf, dass weder der sich verändernde Literaturbetrieb 
noch die konformistisch ausgerichtete Wohlstandsgesellschaft der Wirtschaftswunderjahre für Kreuders Absturz in die Bedeutungslosigkeit ausschlaggebend waren, sondern dessen „autobiographische Inszenierung“ (Rauer 2008b: 245). Trotzdem nahmen die sich verändernden kulturellen und wirtschaftlichen Bedingungen Einfluss auf Kreuders erfolgsbedingtes Selbstverständnis, da er eine Ausbreitung seiner Erkenntnisse und Theoreme zunehmend gefährdet sah. Als elitärer Einzelgänger reklamierte Kreuder in seinen literarischen und essayistischen Beiträgen nach 1945 einen Sonderstatus, der zwar mit Minorität spielt, aber nicht minoritär sein will. Höhepunkt dieser Inszenierung sind der Artikel für Books Abroad und der Roman Herein ohne anzuklopfen. In diesem Roman weist sich der namenlose Protagonist aus Verzweiflung über die Gefühllosigkeit der Masse und aus Unverständnis über die Welt selbst in eine ,Irrenanstalt' ein. Dort trifft er auf eine Gruppe gleichgesinnter ,Kranker“ die sich von ihm bald „Heilung“ erwarten, die durch die Initiierung zu einem eigenverantwortlichen Leben im Sinne der gewohnten romantisch-magischen Vorstellungen Kreuders wie in der Gesellschaft vom Dachboden und den Unauffindbaren ein letztes Mal gelingt. Die dominante Rolle des Protagonisten wird von den Insassen sofort akzeptiert und bis zum Ende nicht hinterfragt, wohl aber von den zahlreichen Akteuren aus dem Literaturbetrieb, die im Roman wie im Books Abroad Artikel namentlich angegriffen oder übergangen werden.

Dieser an sich übliche Vorgang zerstörte die auf Erfolg bauende Autorposition, deren fortlaufender Absturz von Kreuder weiterhin konsequent autobiographisch inszeniert wird. Die Initiationen in Agimos oder die Weltgehilfen (1959) schlagen fehl oder verlieren sich im Diffusen. Der mehr schäbige als geheimnisvolle Männerbund in Hörensagen (1969) schreckt dann nicht einmal mehr vor medizinischen Experimenten mit Inhaftierten zurück, um das Weltbild Kreuders zu verbreiten. Ihre anschließende Flucht scheitert schließlich so ausgiebig in der Provinzheimat Kreuders, dass Hörensagen auch als hessischer Regionalroman gelesen werden könnte. Trotz des hier vorgeführten eigenen Scheiterns ist der Roman doch wegen des Zusammenwirkens der unzuverlässigen Erzähler formal interessant und besticht durch seinen stellenweise galligen Selbsthumor. Dass die Fiktion hier vieles kaschiert, die Lebensbilanz tatsächlich anders ausfiel, wird an einem der letzten Briefe kurz vor Kreuders Tod am Weihnachtstag 1972 deutlich, der sich auf die Verleihung des Nobelpreises an Heinrich Böll bezieht:

„Bei der Fernseh-Nobel-Feier am Sonntag [...] packte es mich tiefer: man hat mir Unrecht getan. Ich hab gegeben, was wenige konnten, mit Müh und Geduld, in meinen vergriffenen Büchern. War mir arg. Mir wurde bang. Nächstes Jahr 70, und man wird nicht erwähnt, man soll noch immer Brötchen verdienen müssen." ${ }^{13}$

What's wrong with the Nobel Prize?

13 Das Zitat findet sich auf der Webseite des Ernst Kreuder Freundeskreises unter der Spitzmarke Kreuder entdecken? [http://www.ernst-kreuder.de/frameset.htm?frame=http\%3A//www.ernst-kreuder.de/einstieg. htm] (20. 8. 2020). 


\section{Quellen und Literatur}

Andersch, Alfred (1974): Scheinwerfer auf einen Seiltänzer. In: Stoll, Christoph - Goldmann, Bernd (hrsg.): Ernst Kreuder. Von ihm. Über ihn. Mainz: Akademie der Wissenschaften und der Literatur, S. 38-39.

Brenner, Peter J. (1997): Nachkriegsliteratur. In: Glaser, Horst A. (hrsg.): Deutsche Literatur zwischen 1945 und 1995. Bern [et al.]: Haupt, S. 33-57.

Deschner, Karlheinz (1973): Ein Opfer der Gruppe 47. In: Konkret 19, No. 4, S. 42-44.

Egyptien, Jürgen (2006): Einführung in die deutschsprachige Literatur seit 1945. Darmstadt: WBG.

Fiedler, Peter-Alexander (1990): Träumer und Rebell. Nachwort. In: Ders. (hrsg.): Ernst Kreuder: Die Gesellschaft vom Dachboden. Erzählungen, Essays, Selbstaussagen. Berlin - Weimar: Aufbau, S. 607-634.

Haberland, Detlef (2017): Brüche und Kontinuitäten. Ein Plädoyer für eine Literaturgeschichte der Einzelanalyse. In: Ders. (hrsg.): Ästhetik und Ideologie 1945. Wandlung und Kontinuität poetologischer Paradigmen deutschsprachiger Schriftsteller. München: De Gruyter Oldenbourg, S. 11-36.

Hocke, Gustav René (1946): Deutsche Kalligraphie oder Glanz und Elend der modernen Literatur. In: Der Ruf 1, Nr. 7, S. 9-10.

Hubig, Christoph (2010): Kulturbegriff - Abgrenzungen, Leitdifferenzen, Perspektiven. In: Banse, Gerhard - Grunwald, Armin (hrsg.): Technik und Kultur. Bedingungs- und Beeinflussungsverhältnisse. Karlsruhe: KIT Scientific Publishing, S. 55-71.

Ivask, Ivan (1984): Ernst Erich Noth (1909-1983). In: World Literature Today 58, No. 1, S. 14.

Krappmann, Jörg (2017): Dichterpriestertum als Erfolgsgarantie? Ernst Kreuders poetologisches Konzept zwischen den Zeiten. In: Haberland, Detlef (hrsg.): Ästhetik und Ideologie 1945. Wandlung und Kontinuität poetologischer Paradigmen deutschsprachiger Schriftsteller. München: De Gruyter Oldenbourg, S. 289-300.

Krappmann, Jörg (2013): Magischer Realismus. In: Brittnacher, Hans Richard - May, Markus (hrsg.): Phantastik. Ein interdisziplinäres Handbuch. Stuttgart: Metzler, S. 529-537.

Kreuder, Ernst (1971): Durchgehend geöffnet. In: Salis, Richard (hrsg.): Motive. Deutsche Autoren zur Frage: Warum schreiben Sie? Tübingen - Basel: Erdmann.

Kreuder, Ernst (1951): Antwort auf die Rundfrage What's Wrong with the Nobel Prize? In: Books Abroad 25, No. 2, S. 115-116.

Kreuder, Ernst (1951): Elisabeth Langgässer. In: Books Abroad 25, No. 1, S. 4-6.

Kreuder, Ernst (1950): Nachruf auf Elisabeth Langgässer. In: Jahrbuch der Akademie der Wissenschaften und der Literatur. Mainz, S. 143-147.

Kreuder, Ernst - Mumm, Carl (1954): A Quarter Century of German Literature. In: Books Abroad 28, No. 1, S. 4-14.

Lederer, Max (1947): Rezension zu Deutsche Innere Emigration. Anti-Nationalsozialistische Zeugnisse aus Deutschland. In: Books Abroad 21, No. 1, S. 91-92.

Lindner, Martin (1994): Leben in der Krise. Zeitromane der Neuen Sachlichkeit und die intellektuelle Mentalität der klassischen Moderne. Stuttgart: Metzler.

Martini, Fritz (1958): Deutsche Literaturgeschichte von den Anfängen bis zur Gegenwart (9. Aufl.). Stuttgart: Kröner.

Mayer, Elizabeth M. (1948): Rezension zu Bekenntnis zu Ernst Wiechert. Ein Gedächtnisbuch zum 60. Geburtstag des Dichters. In: Books Abroad 22, No. 2, S. 191. 
Meier, Uwe (1999): Ernst Erich Noth. In: Neue Deutsche Biographie 19 (1999), S. 358-359.

Müller, Gustav (1947): Rezension zu Werner Bergengruen: Die Sultansrose und andere Erzählungen. In: Books Abroad 21, No. 4, S. 456.

Nicklaus, Kirsten (2001): Abendland und Wirtschaftswunder. Zur kulturkritischen Physiognomie der westdeutschen Romanprosa zwischen 1945 und 1959. Dissertation [masch.]. https://macau.uni-kiel.de/receive/diss_mods_00000633 (11. 8. 2020).

Noth, Ernst Erich (2009): Erinnerungen eines Deutschen. Erstes Buch: Die deutschen Jahre. Frankfurt a. M.: Glotzi.

Noth, Ernst Erich (1950): Rezension zu Ernst Kreuder: Die Unauffindbaren. In: Books Abroad 24, No.1, S. 33.

Noth, Ernst Erich (1949): Rezension zu Thomas Mann: Joseph und seine Brüder. In: Books Abroad 23, No. 4, S. 365-366.

Puknus, Heinz (1982): Ernst Kreuder. In: Kritisches Lexikon zur deutschsprachigen Gegenwartsliteratur (KLG). München: Edition Text + Kritik. S. 1-11.

Pusey III., William W. (1947): Rezension zu Ernst Wiechert: Der Totenwald. In: Books Abroad 21, No. 1, S. 46-47.

Rauer, Stephan (2008a): „BUTKU“. Zu Ernst Kreuders Kurzgeschichten im Dritten Reich. In: Würmann, Carsten - Warner, Ansgar (hrsg.): Im Pausenraum des Dritten Reichs. Zur Populärkultur im nationalsozialistischen Deutschland. Bern: Lang, S. 229-245.

Rauer, Stephan (2008b): Ernst Kreuder. Vorgeführtes Erzählen - Vorgeführtes Erinnern. (19331959). Bielefeld: Aisthesis.

Richter, Hans Werner (1947): Literatur im Interregnum. In: Der Ruf 15, S. 10-11.

Schalk, Axel (1997): Die Bundesrepublik im Roman nach 1945. In: Glaser, Horst A. (hrsg.): Deutsche Literatur zwischen 1945 und 1995. Bern [et al.]: Haupt, S. 235-247.

Schulz, Christoph (1992): „Macht die Augen auf und träumt!“. Ernst Kreuders erzählerisches Werk. Frankfurt a. M. [et al.]: Lang.

Stach, Reiner (1996): Stil, Motiv und fixe Idee. Über einige Untiefen der Jahnn-Lektüre. In: Böhme, Hartmut - Schweikert, Uwe (hrsg.): Archaische Moderne. Der Dichter, Architekt und Orgelbauer Hans Henny Jahnn. Stuttgart: M \& P, S. 346-361.

Strelka, Joseph Peter (1989): Ernst Erich Noth. In: Spalek, John M. - Strelka, Joseph Peter (hrsg.): Deutschsprachige Exilliteratur seit 1933. Band 2, Teil 1. Bern: Francke, S. 725-736.

Williams, Rhys W. (1991): Deutsche Literatur in der Entscheidung. Alfred Andersch und die Anfänge der Gruppe 47. In: Fetscher, Justus - Lämmert, Eberhard - Schutte, Jürgen (hrsg.): Die Gruppe 47 in der Geschichte der Bundesrepublik. Würzburg: Königshausen \& Neumann, S. 23-43.

Windfuhr, Manfred (2018): Zukunftsvisionen. Von christlichen, grünen und sozialistischen Paradiesen und Apokalypsen. Bielefeld: Aisthesis.

Žmegač, Viktor hrsg. (1984): Geschichte der deutschen Literatur vom 18. Jahrhundert bis zur Gegenwart. Band III 1918-1980. Königstein/Ts.: Athenäum. 
Stiftungsprofessor Doc. Mgr. Jörg Krappmann, Ph.D. / krappmann@centrum.cz

Univerzita Palackého v Olomouci, Filozofická fakulta, Katedra germanistiky

Křížkovského 10, 77900 Olomouc, CZ

This work can be used in accordance with the Creative Commons BY-SA 4.0 International license terms and conditions (https://creativecommons.org/licenses/by-sa/4.0/legalcode). This does not apply to works or elements (such as image or photographs) that are used in the work under a contractual license or exception or limitation to relevant rights 
